\title{
Caractérisation phénotypique des ressources génétiques porcines des départements de l'Ouémé et du Plateau au Bénin
}

\author{
Issaka Youssao Abdou Karim ${ }^{1 *}$ Ignace Ogoudanan Dotché ${ }^{1}$ \\ Soumanou Seibou Toleba ${ }^{2}$ Kevin Sagui Kassa ${ }^{1}$ \\ Serge Gbênagnon Ahounou ${ }^{1}$ Chakirath Salifou ${ }^{1}$ \\ Mahamadou Dahouda ${ }^{2}$ Nicolas Antoine-Moussiaux ${ }^{3}$ \\ Jean-Paul Dehoux ${ }^{4}$ Guy Apollinaire Mensah ${ }^{5}$
}

\begin{abstract}
Mots-clés
Porcin, phénotype, race indigène, croisement, mensuration corporelle, Bénin
\end{abstract}

Submitted: 12 July 2017

Accepted: 2 May 2018

Published: 9 July 2018

DOI: $10.19182 /$ remvt.31219

\section{Résumé}

L'élevage porcin est très pratiqué au Sud-Bénin et implique une diversité de races ou de populations. L'objectif de l'étude était de caractériser les différents porcs sur le plan morphométrique et phénotypique. Ainsi, les données phénotypiques ont été collectées sur 149 porcs, dont 14 améliorés, 91 croisés et 44 locaux. Les porcs de type génétique local ont présenté des mesures morphologiques significativement inférieures $(p<0,05)$ à celles des porcs améliorés et des animaux issus des croisements entre les porcs améliorés et les porcs locaux. Les poils des porcs locaux étaient significativement plus courts $(p<0,05)$ que ceux des porcs améliorés et des croisés. La couleur de la robe a varié d'un type génétique à l'autre. La couleur la plus rencontrée a été le blanc uniforme, suivi du noir uniforme chez tous les types génétiques. Le profil de la tête était plus rectiligne chez les porcs locaux, et plus concave chez les porcs améliorés et chez les croisés. Les oreilles dressées étaient moins observées chez les croisés. Elles étaient orientées vers l'avant chez les porcs améliorés et chez les croisés alors qu'elles étaient dressées et orientées vers l'arrière chez les porcs locaux. La queue en tire-bouchon a été significativement $(p<0,05)$ plus présente chez les porcs améliorés que chez les porcs croisés, et elle a été plus présente chez ces derniers que chez les porcs locaux. La ligne dorsale droite a été davantage observée chez les porcs locaux et chez les croisés que chez les porcs améliorés chez lesquels la ligne était plus creuse. Les porcs croisés ont présenté une grande similarité avec les porcs améliorés.

- Comment citer cet article : Youssao Abdou Karim I., Dotché I.O., Seibou Toleba S., Kassa K.S., Ahounou S.G., Salifou C., Dahouda M., Antoine-Moussiaux N., Dehoux J.-P., Mensah G.A., 2018. Phenotypic characterization of pig genetic resources in the departments of Oueme and Plateau in Benin. Rev. Elev. Med. Vet. Pays Trop., 71 (1-2): 59-65, doi: 10.19182/remvt.31219
1. Département de production et santé animales, Ecole polytechnique d'Abomey-Calavi, Université d'Abomey-Calavi, 01 BP 2009, Cotonou, Bénin. 2. Département de production animale, Faculté des sciences agronomiques, Université d'Abomey-Calavi, Cotonou, Bénin.

3. Département de production animale, Faculté de médecine vétérinaire, Université de Liège, B-4000 Liege, Belgique.

4. Université catholique de Louvain (UCL), 1200 Bruxelles, Belgique.

5. Institut national de recherches agricoles du Bénin (INRAB), Cotonou 01, Bénin.

* Auteur pour la correspondance

Tél. : +22995285988; email : iyoussao@yahoo.fr

\section{INTRODUCTION}

Le cheptel porcin était estimé à 414000 têtes en 2013 au Bénin, apportant une part non négligeable pour la couverture de la sécurité alimentaire, surtout des populations du sud du pays (Direction de l'élevage, 2015). Il apporte 4968 tonnes de viande par an au pays (Countrystat, 2017). Cette production nationale en viande porcine n'arrive pas à couvrir les besoins des consommateurs et le déficit est importé des pays limitrophes. La conséquence directe de ces importations est l'introduction de nouvelles pathologies dont la plus importante est la peste porcine africaine au Bénin (Direction de l'élevage, 2015). Pour 
limiter ces conséquences, la production nationale doit être améliorée sur la base des caractéristiques et des aptitudes de chacune des races ou des populations. Pour ce faire, la caractérisation phénotypique et génétique est une nécessité pour une meilleure connaissance des ressources zoogénétiques, de leurs utilisations actuelles et éventuellement futures pour l'alimentation (FAO, 2012). Cette caractérisation permettra de faire des prédictions objectives et fiables sur les performances des animaux en fonction des systèmes de production. Ces systèmes ont été décrits dans de nombreux travaux (Houndonougbo et al., 2012 ; Oluwole et al., 2014 ; Alenyorege et al., 2015). En revanche, sur le plan morphométrique, aucun travail n'a été réalisé pour caractériser les porcs au Bénin en dehors des mensurations sur la hauteur au garrot, le périmètre thoracique et la longueur scapulo-ischiale de la race porcine locale (Youssao et al., 2008). Sur le plan génétique, les travaux ont porté sur la caractérisation moléculaire et sur l'amélioration des performances zootechniques par croisements entre la race locale et les races exotiques (Ramirez et al., 2009 ; Youssao et al., 2009 ; Amills et al., 2013). Dans les exploitations porcines du Sud-Bénin, les croisements effectués aujourd'hui par les éleveurs ont engendré une diversité de types génétiques dont les caractéristiques sont peu connues. L'objectif de l'étude a été de caractériser les porcs élevés au Bénin sur la base des mesures morphométriques et des caractères phénotypiques afin de favoriser la prise de décision sur les priorités à mettre en œuvre pour la mise en valeur des ressources génétiques porcines béninoises.

\section{MATERIEL ET METHODES}

\section{Cadre de l'étude}

L'étude a été réalisée dans les départements de l'Ouémé et du Plateau situés au Sud-Bénin (figure 1). Le département de l'Ouémé est situé entre $6^{\circ} 40^{\prime} \mathrm{N}$ et $2^{\circ} 30^{\prime} \mathrm{E}$ et couvre une superficie de 1281 kilomètres carrés avec une population de 1100404 habitants en 2013 (INSAE, 2015). Les religions pratiquées dans ce département sont le christianisme $(75,8 \%)$, l'islam $(12,1 \%)$ et le vodoun $(0,6 \%)$ (INSAE, 2015). Le département du Plateau est compris entre $7^{\circ} 10^{\prime} \mathrm{N}$ et $2^{\circ} 34^{\prime}$ E et couvre une superficie de 3264 kilomètres carrés, soit environ $3 \%$ du territoire national pour une population totale de 622372 habitants (INSAE, 2015). Les religions pratiquées dans ce département sont le christianisme $(59,8 \%)$, l'islam $(18,6 \%)$ et le vodoun (3,3\%) (INSAE, 2015).

\section{Animaux et mode d'élevage}

Les animaux étudiés étaient des porcs issus de races porcines élevées dans les départements de l'Ouémé et du Plateau. Les données ont été relevées sur 149 porcs, dont 14 porcs améliorés (12 femelles et 2 mâles), 91 porcs croisés (12 mâles et 79 femelles) et 44 porcs locaux (5 mâles et 39 femelles) dans les deux départements. Les porcs améliorés regroupaient des animaux de races exotiques et des croisés entre les races exotiques, alors que les animaux qualifiés de croisés étaient les produits issus de croisements entre les porcs améliorés et les porcs locaux. Les probables races exotiques impliquées dans les croisements étaient les porcs Large White, Landrace et Duroc importés le plus souvent du Nigeria par les éleveurs. De plus, certains éleveurs se sont procuré par le passé des animaux Large White et Landrace à la ferme d'élevage de Kpinnou (ferme d'Etat où les porcs Large White, Landrace et Meishan avaient été importés et diffusés dans les élevages).

Les animaux étaient issus de 50 élevages en claustration permanente, situés dans diverses communes : dans le département de l'Ouémé, 16 à Porto-Novo, 10 à Sème-Podji, 5 à Adjarra, 4 à Akpro-Misérété et 1 à Avrankou ; et dans le département du Plateau, 9 à Pobè, 2 à Adja-Ouèrè, 2 à Ifangni et 1 à Sakété (figure 1). L'aliment et l'eau étaient servis une ou deux fois par jour selon la disponibilité de l'éleveur. Il s'agissait en général de drêches de brasserie, de sons de céréales et d'oléagineux (maïs, soja, etc.), de tourteau de palmiste, de résidus de récolte (épluchures de manioc, noix de palme, etc.), de verdure (feuille de bananier, feuille de manioc, feuille de papayer, etc.), de restes de cuisine issus des ménages et quelquefois de compléments en vitamines et minéraux.

Sur le plan sanitaire, la prophylaxie était basée sur l'entretien des loges, le déparasitage interne et la lutte contre les ectoparasites. En ce qui concerne le volet reproduction, les géniteurs ont été choisis par les éleveurs en fonction des critères de sélection qui leur ont semblé importants. Certains les choisissaient dans d'autres élevages sans tenir compte de critères de sélection. Les critères de sélection souvent utilisés étaient le nombre de tétines, la taille de la portée, le nombre de nés vivants, l'absence de défauts génétiques pour les femelles et la conformation et le développement testiculaire pour les mâles. Etant au contact des animaux, les éleveurs reconnaissaient aisément les œstrus et le verrat était alors présenté à la truie pour la saillie. Pour tous les éleveurs rencontrés, le diagnostic de gestation était essentiellement basé sur le non-retour des chaleurs trois semaines après la saillie.

\section{Phénotypage}

La méthodologie utilisée pour la collecte des données a été celle de l'enquête rétrospective par entretien avec l'éleveur, suivie des mesures corporelles sur les animaux des types génétiques identifiés. Les éleveurs ont été choisis suivant les critères d'accessibilité et de leur disponibilité à fournir les informations. Les types génétiques élevés ont ainsi été repérés et décrits. L'enquête a permis d'identifier les types génétiques utilisés par les éleveurs (selon leurs dires, l'utilisation et l'origine des animaux), de déterminer le statut (gestante / non gestante) et le rang de mise bas des femelles. Pour la description, deux fiches de renseignements ont été utilisées. La première renseignait sur les caractères phénotypiques qualitatifs (motif et couleur de la robe, forme du
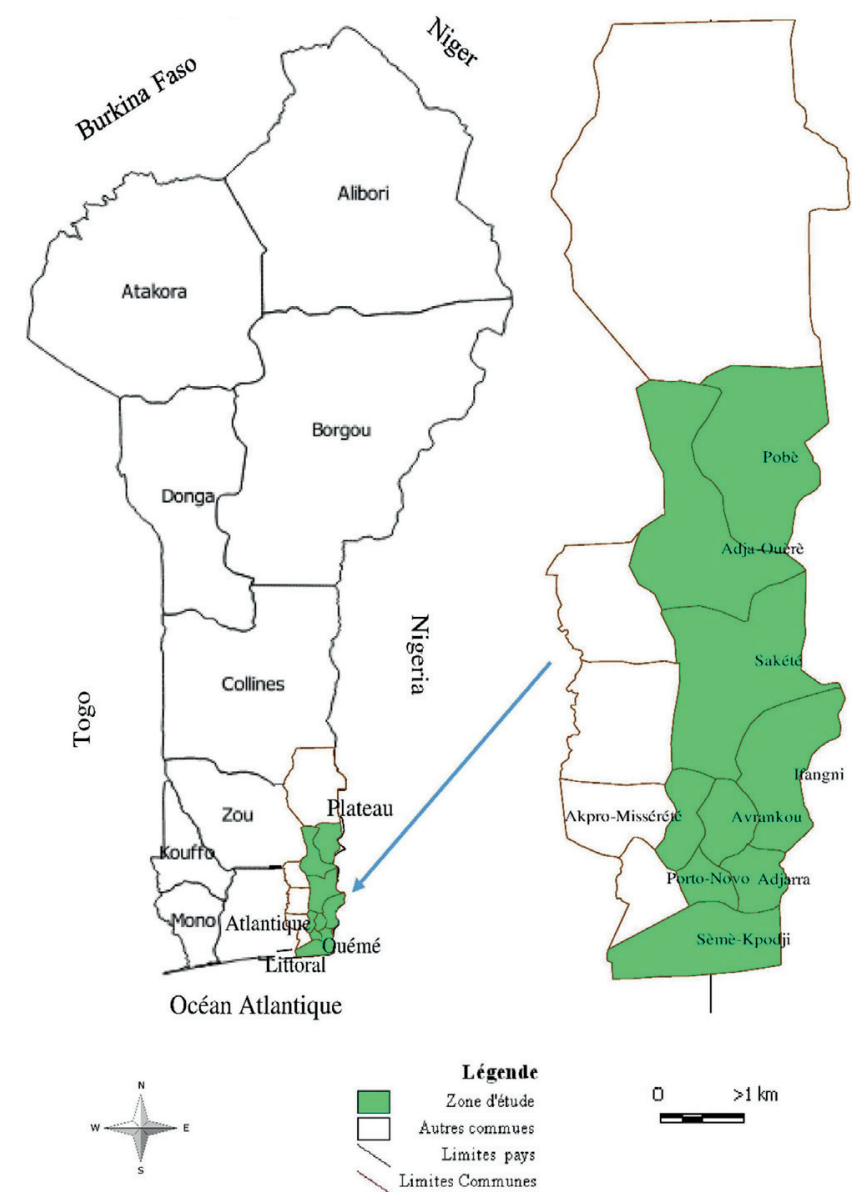

Figure 1 : zone d'étude sur la caractérisation des porcs au SudBénin. 
museau, forme des poils, etc.) et la seconde était utilisée pour collecter les différentes mesures corporelles (nombre de trayons, longueur des oreilles, longueur de la tête, longueur du museau, périmètre thoracique, etc.). La collecte des données a concerné uniquement les truies vides ayant mis bas au moins deux fois et les verrats reproducteurs.

Les porcs ont été décrits phénotypiquement et les mesures corporelles suivantes ont été relevées à l'aide d'un mètre métrique : longueur de la tête (distance entre la nuque et le bout du museau) ; longueur des oreilles (de la base à l'extrémité inférieure) ; longueur du bassin (distance entre pointe des hanches et pointe des fesses) ; largeur aux hanches (distance entre les deux pointes des hanches) ; périmètre thoracique (en passant verticalement en arrière du garrot et au niveau du passage de sangle) ; hauteur au garrot (distance du sommet du garrot au sol) ; longueur de la queue (distance entre le point d'attachement de la queue jusqu'à l'extrémité) ; dessus de l'épaule (distance entre les deux omoplates) ; circonférence du jarret (pourtour du jarret).

\section{Analyses statistiques}

Après l'encodage, les données ont été analysées avec le logiciel SAS (Cary, NC, USA, 2006). Pour les variables quantitatives (longueur scapulo-ischiale, périmètre thoracique, circonférence du cou, hauteur au garrot, dessus de l'épaule, largeur à la hanche, etc.), la procédure Proc univariate a d'abord été utilisée pour vérifier que les variables suivaient une distribution normale. Un modèle linéaire à effet fixe a ensuite été utilisé pour l'analyse de variance de ces variables quantitatives. Les effets fixes de ce modèle ont été le type génétique et le sexe. L'effet sexe et l'interaction entre le type génétique et le sexe n'ont pas été significatifs et ont été enlevés du modèle d'analyse de variance. L'expression mathématique du modèle final retenu se présente de la manière suivante :

$\mathrm{Y}_{\mathrm{ij}}=\mu+\mathrm{TG}_{\mathrm{i}}+\mathrm{e}_{\mathrm{ij}}$

où $Y_{i j}$ est une mesure corporelle de l'animal $j$, de type génétique $i, \mu$ la moyenne générale, $\mathrm{TG}_{\mathrm{i}}$ l'effet fixe du type génétique i (local, amélioré ou croisé), et $\mathrm{e}_{\mathrm{ij}}$ l'erreur résiduelle aléatoire.

La procédure Proc GLM a été utilisée pour l'analyse de variance et le test de Fisher a été utilisé pour déterminer la significativité de l'effet type génétique sur les variables considérées. Les comparaisons entre les moyennes ont été faites deux à deux par le test $t$ de Student. La procédure Proc princomp a été ensuite utilisée pour l'analyse en composante principale (ACP) des mesures corporelles et des trois types génétiques.

Pour les variables qualitatives, la procédure Proc corresp a été utilisée pour l'analyse factorielle des correspondances (AFC). Les variables prises en compte ont été la race utilisée et les caractères qualitatifs. Une classification ascendante hiérarchique sur la base des coordonnées des 149 porcs sur les composantes de l'AFC les plus significatives a ensuite été réalisée.

Les fréquences ont été calculées pour les variables qualitatives (motif de la robe, couleur de la robe, type d'oreille, forme du museau, etc.) par la procédure Proc freq du logiciel SAS (2006) et les comparaisons ont été faites par le test bilatéral de $\mathrm{Z}$. Pour chaque fréquence relative, un intervalle de confiance $(I C)$ à $95 \%$ a été calculé suivant la formule :

$$
I C=1,96 \sqrt{\frac{[P(1-P)]}{N}}
$$

où $P$ est la fréquence relative et $N$ la taille de l'échantillon.

\section{RESULTATS}

Cette étude a permis de caractériser les trois types génétiques porcins élevés dans les départements de l'Ouémé et du Plateau au Bénin.

\section{Type génétique amélioré}

Le type génétique amélioré est issu de divers croisements entre les races exotiques pures. En moyenne cet animal était long de 79,1 cm avec un bassin long de $23,1 \mathrm{~cm}$ et une tête longue de $31,4 \mathrm{~cm}$. La figure 2A présente un verrat amélioré. Les poils (soies) étaient en majorité longs et la robe était en majorité de couleur blanche avec des motifs variés. Les motifs rencontrés étaient unis (42,9\%), tachetés ou pie. La tête présentait un profil généralement concave et se terminait par un museau court et cylindrique de circonférence moyenne de $37,4 \mathrm{~cm}$ (tableaux I et II). En moyenne le cou mesurait 74,5 cm de circonférence et la longueur des oreilles était de 19,3 cm. Ces dernières étaient en majorité dressées et orientées vers l'avant. L'animal

Tableau I

Effet du type génétique sur les caractères morphologiques de trois types de porcins dans le Sud-Bénin

\begin{tabular}{|c|c|c|c|c|c|c|c|}
\hline \multirow[t]{2}{*}{ Variable } & \multicolumn{2}{|c|}{$\begin{array}{l}\text { Porc amélioré } \\
\quad(n=14)\end{array}$} & \multicolumn{2}{|c|}{$\begin{array}{l}\text { Porc croisé } \\
(\mathrm{n}=91)\end{array}$} & \multicolumn{2}{|c|}{$\begin{array}{l}\text { Porc local } \\
(n=44)\end{array}$} & \multirow[t]{2}{*}{$\begin{array}{c}\text { Analyse } \\
\text { de variance }\end{array}$} \\
\hline & Moyenne & DS & Moyenne & DS & Moyenne & DS & \\
\hline Longueur scapulo-ischiale (cm) & $79,1^{a}$ & 11,7 & $74,8^{a}$ & 11,3 & $52,2^{b}$ & 10,2 & $* * *$ \\
\hline Périmètre thoracique $(\mathrm{cm})$ & $117,2^{\mathrm{a}}$ & 29,8 & $110,3^{a}$ & 26,4 & $78,8^{b}$ & 16,4 & $* * *$ \\
\hline Circonférence du cou (cm) & $74,5^{\mathrm{a}}$ & 12,9 & $72,2^{\mathrm{a}}$ & 12,9 & $52,6^{b}$ & 11,2 & $* * *$ \\
\hline Hauteur au garrot $(\mathrm{cm})$ & $75,1^{\mathrm{a}}$ & 11,4 & $72,6^{a}$ & 10,8 & $47,3^{b}$ & 8,1 & $* * *$ \\
\hline Dessus de l'épaule (cm) & $26,1^{a}$ & 5,5 & $26,2^{\mathrm{a}}$ & 6,1 & $17,4^{b}$ & 3,1 & $* * *$ \\
\hline Largeur à la hanche (cm) & $19,3^{a}$ & 3,7 & $18,1^{\mathrm{a}}$ & 3,1 & $12,4^{b}$ & 2,1 & $* * *$ \\
\hline Longueur du bassin $(\mathrm{cm})$ & $23,1^{\mathrm{a}}$ & 4,7 & $22^{\mathrm{a}}$ & 3,1 & $16,3^{b}$ & 3,2 & $* * *$ \\
\hline Circonférence du jarret (cm) & $25,1^{\mathrm{a}}$ & 5 & $22,3^{a}$ & 6,8 & $15,3 b$ & 4,8 & $* * *$ \\
\hline Longueur de la tête $(\mathrm{cm})$ & $31,4^{\mathrm{a}}$ & 3,2 & $30,4^{\mathrm{a}}$ & 3,5 & $25,3^{b}$ & 3,6 & $* * *$ \\
\hline Longueur de l'oreille (cm) & $19,3^{\text {a }}$ & 3,3 & $19,2^{a}$ & 3,5 & $10,8^{b}$ & 2 & $* * *$ \\
\hline Circonférence du museau $(\mathrm{cm})$ & $37,4^{\mathrm{a}}$ & 5,3 & $36,1^{\mathrm{a}}$ & 6,5 & $29,2^{b}$ & 6,7 & $* * *$ \\
\hline Longueur de la queue $(\mathrm{cm})$ & $34,1^{a}$ & 7,4 & $33,5^{\mathrm{a}}$ & 10,8 & $21,9^{b}$ & 5,1 & $* * *$ \\
\hline $\mathrm{Nb}$. de trayons chez les cochettes & $12,9^{a}$ & 1,3 & $12,4^{\mathrm{a}}$ & 1,4 & $9,1^{b}$ & 1,4 & $* * *$ \\
\hline
\end{tabular}

DS : déviation standard

a, b Les moyennes sur une même ligne suivies de la même lettre ne diffèrent pas significativement au seuil de $5 \%$; *** $\mathrm{p}<0,001$. 
présentait une peau à l'aspect majoritairement lisse et une ligne dorsale ensellée. La hauteur au garrot du porc amélioré était de 75,1 cm et le périmètre thoracique de $117,2 \mathrm{~cm}$. La largeur à la hanche était de $19,3 \mathrm{~cm}$ et le dessus de l'épaule de 26,1 cm. Les pieds étaient solides et la circonférence du jarret était de $25,1 \mathrm{~cm}$. La queue était en tirebouchon et mesurait $34,1 \mathrm{~cm}$ de long. Le nombre de tétines était de 13 .

\section{Type génétique croisé}

Le porc croisé est un animal issu du croisement entre les porcs exotiques améliorés et les porcs locaux. Il était de grand format et haut de 72,6 cm en moyenne avec un périmètre thoracique de $110,3 \mathrm{~cm}$ environ (figure 2B). La longueur du corps était de $74,8 \mathrm{~cm}$ et celle du bassin de 22,0 cm en moyenne. Les poils étaient en majorité longs. Les poils bouclés, raides et clairsemés étaient également rencontrés. La couleur de la robe était en majorité blanche avec un motif uniforme. La longueur de la tête était de 30,4 cm en moyenne. Le profil facial était droit ou concave et les oreilles étaient en majorité dressées. Ces dernières étaient majoritairement orientées vers l'avant et mesuraient en moyenne 19,2 cm de long. La peau présentait un aspect en majorité lisse et la ligne dorsale était rectiligne (tableau II). Le museau était court et cylindrique chez la majorité des porcs décrits

\section{Tableau II}

Effet du type génétique sur les caractères phénotypiques de trois types de porcins dans le Sud-Bénin

\begin{tabular}{|c|c|c|c|c|c|c|c|}
\hline \multirow[t]{2}{*}{ Variable } & & \multicolumn{2}{|c|}{$\begin{array}{l}\text { Porc amélioré } \\
\quad(n=14)\end{array}$} & \multicolumn{2}{|c|}{$\begin{array}{l}\text { Porc croisé } \\
(\mathrm{n}=91)\end{array}$} & \multicolumn{2}{|c|}{$\begin{array}{l}\text { Porc local } \\
(n=44)\end{array}$} \\
\hline & & $\%$ & IC & $\%$ & IC & $\%$ & IC \\
\hline \multirow[t]{6}{*}{ Poils } & Bouclés & $0^{\mathrm{a}}$ & 0 & $02,2^{a}$ & 03 & $0^{a}$ & 0 \\
\hline & Raides & $07,1^{\mathrm{a}}$ & 13,5 & $02,2^{a}$ & 03 & $0^{a}$ & 0 \\
\hline & Courts & $14,3^{b}$ & 18,3 & $29,7^{b}$ & 09,4 & $61,4^{\mathrm{a}}$ & 14,4 \\
\hline & Longs & $71,4^{\mathrm{a}}$ & 23,7 & $65,9^{a}$ & 09,7 & $36,4^{b}$ & 14,2 \\
\hline & Denses & $42,9^{a}$ & 25,9 & $28,6^{a}$ & 09,3 & $27,3^{a}$ & 13,2 \\
\hline & Clairsemés & $0,0^{a}$ & 0 & $02,2^{a}$ & 03 & $04,5^{a}$ & 06,2 \\
\hline \multirow[t]{2}{*}{ Museau } & Long et mince & $35,7^{\mathrm{a}}$ & 25,1 & $35,2^{a}$ & 09,8 & $50^{\mathrm{a}}$ & 14,8 \\
\hline & Court et cylindrique & $64,3^{a}$ & 25,1 & $64,8^{a}$ & 09,8 & $50^{a}$ & 14,8 \\
\hline \multirow[t]{3}{*}{ Motif de la robe } & Uni & $42,9^{a}$ & 25,9 & $53,8^{a}$ & 10,2 & $61,4^{\mathrm{a}}$ & 14,4 \\
\hline & Pie & $21,4^{\mathrm{a}}$ & 21,5 & $24,2^{\mathrm{a}}$ & 08,8 & $36,4^{\mathrm{a}}$ & 14,2 \\
\hline & Tacheté & $35,7^{a}$ & 25,1 & $23,1^{\text {a }}$ & 08,7 & $02,3^{b}$ & 04,4 \\
\hline \multirow[t]{6}{*}{ Couleur de la robe } & Blanche & $78,6^{a}$ & 21,5 & $80,2^{a}$ & 08,2 & $75^{\mathrm{a}}$ & 12,8 \\
\hline & Noire & $42,9^{a}$ & 25,9 & $45^{\mathrm{a}}$ & 10,2 & $54,5^{\text {a }}$ & 14,7 \\
\hline & Rouge foncé & $07,1^{\mathrm{a}}$ & 13,5 & $01,1^{\mathrm{a}}$ & 02,1 & $0,0^{\mathrm{a}}$ & 0,0 \\
\hline & Rouge clair & $07,1^{\mathrm{a}}$ & 13,5 & $0,0^{a}$ & 0,0 & $0,0^{\mathrm{a}}$ & 0,0 \\
\hline & Fauve & $21,4^{\mathrm{a}}$ & 21,5 & $08,8^{a}$ & 05,8 & $04,5^{b}$ & 06,2 \\
\hline & Grise & $0,0^{\mathrm{a}}$ & 0,0 & $06,6^{a}$ & 05,1 & $0,0^{\mathrm{a}}$ & 0,0 \\
\hline \multirow[t]{3}{*}{ Profil de la tête } & Convexe & $0,0^{\mathrm{a}}$ & 0,0 & $0,0^{\mathrm{a}}$ & 0,0 & $02,3^{a}$ & 04,4 \\
\hline & Droit & $36,3^{b}$ & 9,9 & $50^{b}$ & 26,2 & $72,7^{\mathrm{a}}$ & 13,2 \\
\hline & Concave & $63,7^{\mathrm{a}}$ & 9,9 & $50^{a, b}$ & 26,2 & $25^{b}$ & 12,8 \\
\hline \multirow[t]{4}{*}{ Type d'oreille } & Pendante & $03,3^{a}$ & 03,7 & $0^{\mathrm{a}}$ & 0 & $02,3^{a}$ & 04,4 \\
\hline & Semi-tombante & $02,2^{a}$ & 03 & $07,1^{\mathrm{a}}$ & 13,5 & $02,3^{a}$ & 04,4 \\
\hline & Tombante & $29,7^{a}$ & 09,4 & $0^{b}$ & 0 & $11,4^{\mathrm{a}}$ & 09,4 \\
\hline & Dressée & $67^{b}$ & 09,7 & $85,7^{a, b}$ & 18,3 & $86,4^{\mathrm{a}}$ & 10,1 \\
\hline \multirow[t]{3}{*}{ Orientation de l'oreille } & Vers I'avant & $78,6^{a}$ & 21,5 & $69,2^{\mathrm{a}}$ & 09,5 & $15,9^{b}$ & 10,8 \\
\hline & Vers l'arrière & $07,1^{a, b}$ & 13,5 & $06,6^{b}$ & 05,1 & $27,3^{a}$ & 13,2 \\
\hline & Vers le haut & $14,3 \mathrm{~b}$ & 18,3 & $25,3^{b}$ & 08,9 & $56,8^{a}$ & 14,6 \\
\hline \multirow[t]{2}{*}{ Peau } & Lisse & $57,1^{\mathrm{a}}$ & 25,9 & $57,1^{\mathrm{a}}$ & 10,2 & $43,2^{\mathrm{a}}$ & 14,6 \\
\hline & Ridée & $42,9^{a}$ & 25,9 & $42,9^{a}$ & 10,2 & $54,5^{\mathrm{a}}$ & 14,7 \\
\hline \multirow[t]{2}{*}{ Type de queue } & Droite & $07,1^{\mathrm{c}}$ & 13,5 & $38,5^{b}$ & 10 & $68,2^{a}$ & 13,8 \\
\hline & En tire-bouchon & $92,9^{a}$ & 13,5 & $60,4^{b}$ & 10 & $31,8^{\mathrm{c}}$ & 13,8 \\
\hline \multirow[t]{2}{*}{ Ligne du dos } & Droite & $21,4^{b}$ & 21,5 & $52,7^{\mathrm{a}}$ & 10,3 & $68,2^{a}$ & 13,8 \\
\hline & Ensellée & $78,6^{a}$ & 21,5 & $47,2^{b}$ & 10,3 & $31,8^{b}$ & 13,8 \\
\hline
\end{tabular}

IC : intervalle de confiance

a, b,c Les pourcentages sur une même ligne suivies de la même lettre ne diffèrent pas significativement au seuil de $5 \%$. 
et sa circonférence était en moyenne de $36,1 \mathrm{~cm}$. La circonférence du cou était de 72,2 cm en moyenne et celle du jarret de $22,3 \mathrm{~cm}$. La largeur aux hanches était de $18,1 \mathrm{~cm}$ et le dessus de l'épaule de $26,2 \mathrm{~cm}$. L'animal présentait 12 tétines. La queue était en majorité en tire-bouchon et mesurait en moyenne $33,5 \mathrm{~cm}$ de long. Les mesures corporelles des porcs croisés étaient similaires à celles des porcs améliorés $(\mathrm{p}>0,05)$, et supérieures à celles des porcs locaux $(\mathrm{p}<0,001)$.

\section{Type génétique local}

Les porcs locaux étaient des animaux de petit format (figure $2 \mathrm{C}$ ). En moyenne la longueur du corps était de $52,2 \mathrm{~cm}$ et celle du bassin de 15,3 cm. La ligne du dos était en majorité rectiligne. La largeur aux hanches était en moyenne de 12,4 cm et le dessus de l'épaule de $17,4 \mathrm{~cm}$. Il était haut de $47,3 \mathrm{~cm}$ et avait un périmètre thoracique de $78,8 \mathrm{~cm}$. Les poils étaient en majorité courts et la robe était de couleur blanche ou noire avec des motifs uniformes (tableau II). L'aspect de la peau était en majorité ridé. La tête était longue de $25,3 \mathrm{~cm}$ en moyenne avec un profil facial majoritairement rectiligne. La circonférence du cou était de 52,6 cm en moyenne. Les oreilles étaient en majorité dressées. Ces dernières mesuraient $10,8 \mathrm{~cm}$ et étaient majoritairement
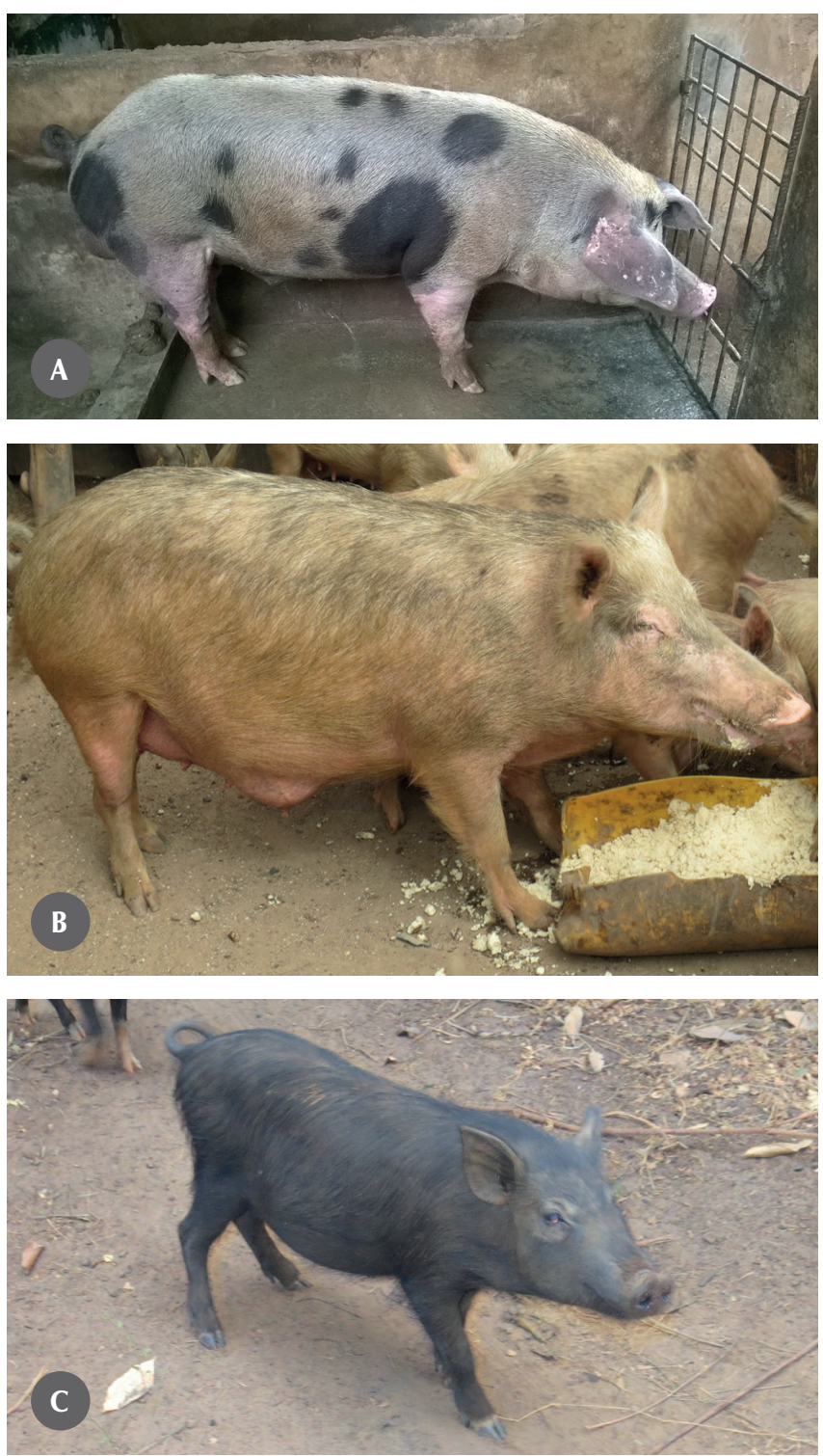

Figure 2 : différents types génétiques de porcs caractérisés au Sud-Bénin. $A$ : verrat amélioré ; $B$ : truie croisée ; $C$ : truie locale. orientées vers le haut. Le museau de l'animal était long et mince ou court et cylindrique avec une circonférence de $29,2 \mathrm{~cm}$. La queue était majoritairement droite et mesurait $21,9 \mathrm{~cm}$. Les porcs locaux présentaient neuf tétines en moyenne.

\section{Comparaison des mesures morphologiques et des caractères phénotypiques entre types génétiques}

Deux axes ont été retenus pour l'interprétation des résultats de l'analyse factorielle des correspondances $\left(\chi^{2}=431,1 ; p<0,001\right)$. La contribution à l'inertie totale des deux axes factoriels a été de $100 \%$ (78,9\% sur l'axe 1 et 20,1\% sur l'axe 2). L'AFC a montré que les caractères comme le profil droit (rectiligne), la robe pie, le museau long et mince, les poils courts, les oreilles dressées orientées vers l'arrière ou le haut caractérisaient les porcs locaux (figure 3). Les porcs améliorés se caractérisaient par une robe blanche tachetée de noir, des poils denses, une queue en tire-bouchon et des oreilles semitombantes (figure 3). Les croisés se distinguaient par un museau court et cylindrique, des poils longs, une peau lisse, des oreilles orientées vers l'avant et une robe grise (figure 3).

L'analyse en composantes principales des mesures morphométriques a permis également de discriminer les trois types génétiques (figure 4). La quasi-totalité des variables était représentée dans l'axe 1 qui expliquait 97,5\% de la variation et l'axe 2 ne représentait que 1,5\% des variations. Les porcs améliorés étaient proches des croisés et représentés dans l'axe 1, alors que les porcs locaux étaient représentés dans

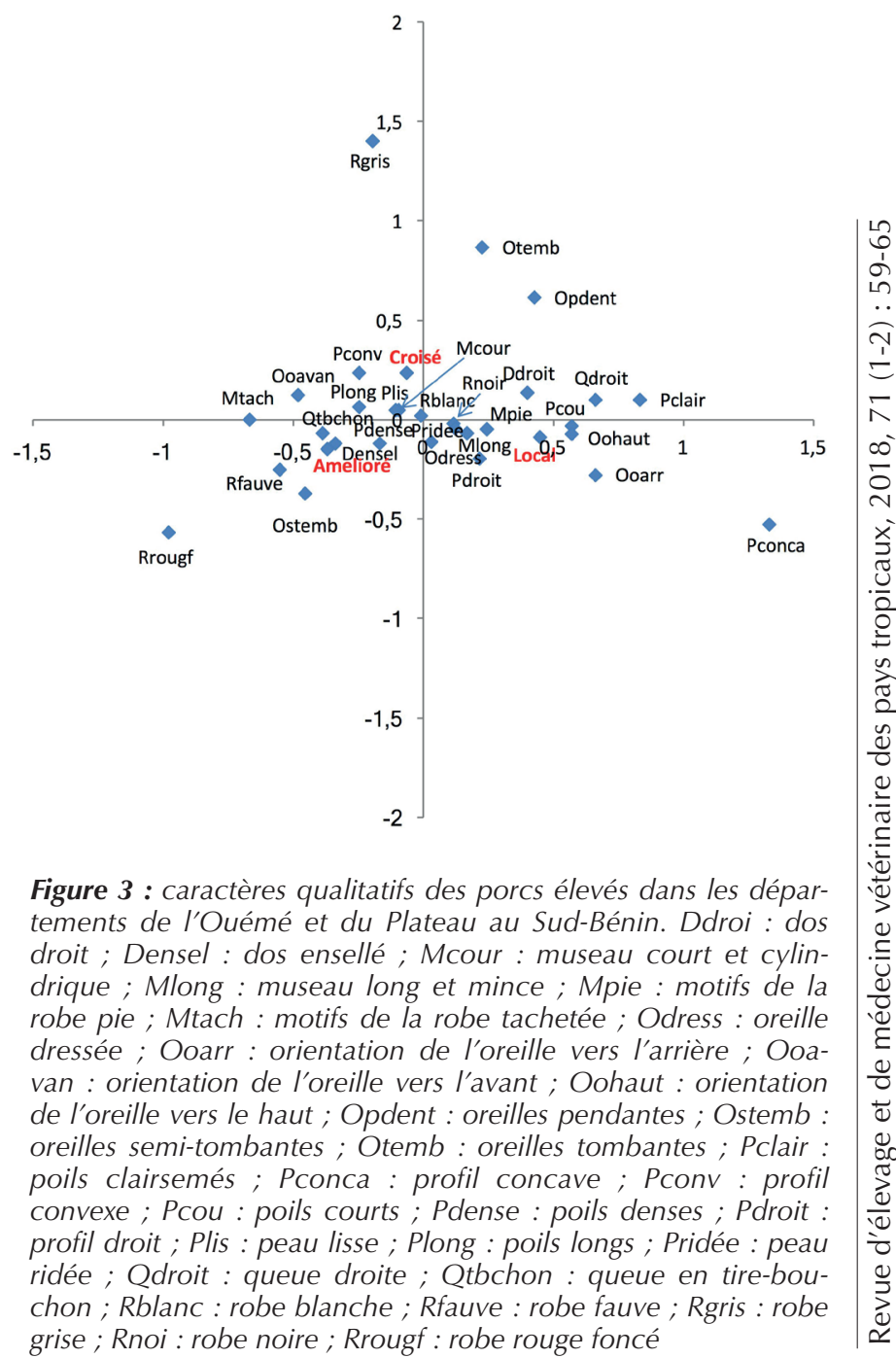




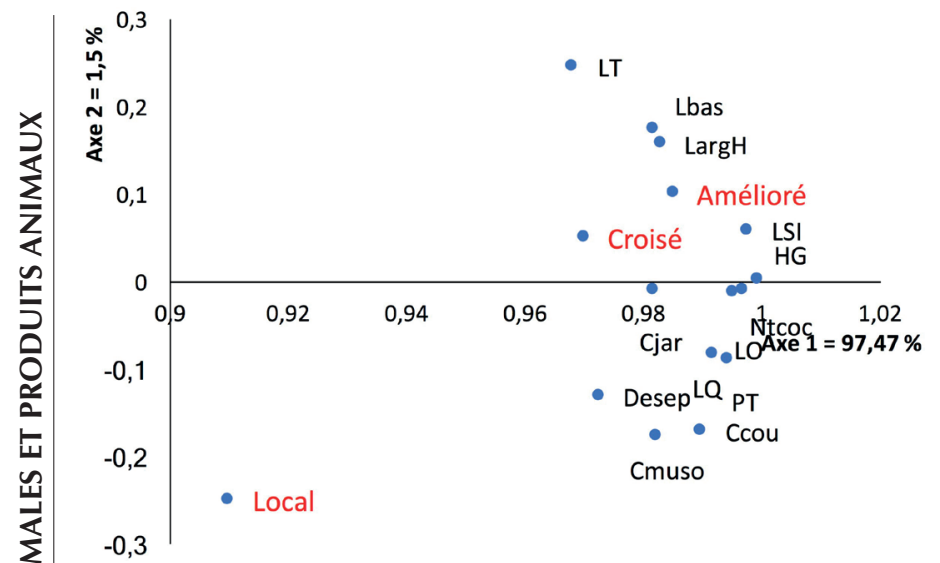

Figure 4 : distribution spatiale des mesures morphométriques des races porcines locale, améliorée et croisée au Sud-Bénin. Ntcoc : nombre de trayons chez les cochettes; LSI : longueur scapulo-ischiale, LO : longueur des oreilles; LT : longueur de la tête ; PT : périmètre thoracique ; Ccou : circonférence du cou ; Cmuso : circonférence du museau ; Local : race locale ; Amelio : race améliorée ; Croisé : amélioré x local ; HG : hauteur au garrot; $L Q$ : longueur de la queue; Desep : dessus de l'épaule ; LargH : largeur à la hanche ; LBas : longueur du bassin ; CJar : circonférence du jarret.

l'axe 2. Le type génétique local a présenté des mesures morphologiques inférieures $(\mathrm{p}<0,001)$ à celles du type génétique amélioré et des produits issus des croisements entre les types génétiques améliorés et les porcs locaux (tableau II).

Les poils des porcs locaux ont été plus courts $(\mathrm{p}<0,05)$ que ceux des types améliorés et des croisés (tableau II). Les poils clairsemés ont été observés seulement chez les porcs locaux et les croisés, alors que les poils raides ont été décrits chez les porcs améliorés et les croisés. La couleur de la robe a peu varié d'un type à l'autre, excepté la couleur fauve qui a été davantage rencontrée chez les porcs améliorés et les croisés. La robe grise n'a été présente que chez les croisés alors que la couleur rouge clair a été décrite uniquement chez les porcs améliorés. Le motif de la robe n'a pas varié d'un type génétique à l'autre pour les robes pie et uniformes. En revanche, la robe tachetée a été davantage rencontrée chez les porcs améliorés et les croisés que chez les porcs locaux. La forme du museau n'a pas varié selon le type génétique, la majorité des porcs avaient un museau court et cylindrique. La seconde forme décrite était le museau long et mince et la moitié des porcs locaux présentaient cette forme de museau. Le profil de la tête des porcs locaux a été plus rectiligne $(\mathrm{p}<0,05)$ que ceux des porcs améliorés et des croisés plus concaves. Les oreilles dressées étaient moins observées chez les porcs améliorés. Les oreilles étaient orientées vers l'avant chez les porcs améliorés et les croisés alors qu'elles étaient orientées vers le haut chez les porcs locaux (tableau II). Le type de queue a varié d'un type à l'autre. La proportion de porcs améliorés présentant des queues en tirebouchon a été supérieure $(\mathrm{p}<0,05)$ à celle des porcs croisés, qui a été elle-même supérieure à celle des porcs locaux. La ligne dorsale droite a été davantage observée chez les porcs locaux et croisés que chez les porcs améliorés chez lesquels la ligne était plus creusée. Les croisés ont présenté une grande similarité avec la race améliorée.

\section{- DISCUSSION}

La présence de porcs locaux, améliorés ou croisés au Sud-Bénin a déjà été signalée (Youssao et al., 2008 ; Houndonougbo et al., 2012). Le type génétique local a présenté des caractéristiques similaires à celles décrites pour les porcs locaux africains (Meyer, 2016) sauf les poils qui étaient courts alors qu'ils ont été décrits comme étant longs chez les porcs locaux africains (Meyer, 2016). Les poils denses, clairsemés et longs observés chez certains porcs locaux ont déjà été décrits au Ghana chez le porc local Ashanti, mais sur différentes parties du corps de l'animal (Alenyorege et al., 2015). Ainsi, les poils denses ont été décrits sur le dos de l'animal et les poils clairsemés sur les côtés (Alenyorege et al., 2015). Les porcs locaux ont présenté des mesures morphologiques plus faibles. Des résultats similaires ont été rapportés au Nigeria où le porc indigène présente des mesures morphologiques plus faibles que les croisés (Adeola et al., 2013). De plus, les porcs ont présenté des mesures supérieures à celles des porcs rapportés par Adeola et al. (2013). Par ailleurs, les mâles des porcs croisés (local et Large White) du Nigeria ont présenté de la naissance au sevrage une longueur du corps, un périmètre thoracique et une circonférence de la croupe plus élevés que ceux des femelles, alors que les femelles ont présenté des mesures, comme la longueur de l'oreille et la hauteur au garrot, plus élevées que les mâles (Oluwole et al., 2014). Cet effet sexe n'a pas été mis en évidence dans la présente étude compte tenu de la rareté des mâles puisqu'elle portait sur les adultes, alors que ces auteurs ont travaillé sur les jeunes. Selon les mêmes auteurs, la corrélation entre le poids et les mesures corporelles montre que ces dernières peuvent servir à sélectionner les animaux selon le poids. Les porcs croisés ont présenté une grande similarité avec la race améliorée du point de vue phénotypique. Le croisement entre les porcs de type génétique amélioré et local devrait donner des sujets intermédiaires.

Dans cette logique classique, Youssao et al. (2009) obtiennent dans une étude réalisée au sud du Bénin les résultats suivants : le poids des porcelets Large White est le triple de celui des porcelets locaux et les croisés ont un poids intermédiaire et significativement différent de ceux des parentaux $(\mathrm{p}<0,001)$; l'effet hétérosis est en moyenne de $27 \%$ chez les mâles et de $17 \%$ chez les femelles issues du croisement entre mâle Large White et femelle locale. Dans le cas du croisement mâle local et femelle Large White, l'effet hétérosis moyen du poids est de $18,3 \%$ chez les mâles et de 5,3\% chez les femelles.

L'absence de différence significative entre les croisés et les améliorés pourrait s'expliquer par deux hypothèses : a) les croisés ont bénéficié d'une double hétérosis, individuelle parce qu'ils proviennent d'un croisement entre deux races différentes, et paternelle parce que le père est issu d'un produit de croisement; b) les résultats de la présente étude ont été obtenus sur des animaux adultes qui ont fini leur croissance, alors que Youssao et al. (2009) ont travaillé sur des porcelets. Une différence entre les croisés et les améliorés aurait pu être observée si les travaux avaient été réalisés sur des porcs en croissance dans la présente étude. Enfin, l'absence de différence entre les croisés et les améliorés pourrait être liée à l'origine des types génétiques améliorés, à la diversité des races impliquées dans les différents croisements et à l'adaptation des porcs améliorés aux facteurs d'environnement.

\section{- CONCLUSION}

Leétude a permis de décrire phénotypiquement trois types génétiques porcins (améliorés, croisés et locaux) élevés dans les départements de l'Ouémé et du Plateau. Les porcs locaux ont montré de plus faibles mesures morphologiques. Les porcs améliorés et les croisés avaient des caractéristiques similaires. Certains porcs croisés ont présenté des poils bouclés et clairsemés, ainsi que des oreilles tombantes et pendantes, contrairement aux porcs améliorés. L'étude de la structure génétique de ces populations permettrait de les comparer à un panel de populations porcines déjà génotypées et représentatives de la diversité génétique mondiale.

\section{Remerciements}

Les auteurs remercient l'Académie de recherche et d'enseignement supérieur (ARES) de la Commission de la coopération au 
développement $(\mathrm{CCD})$ de la Belgique pour son appui à la réalisation de ces travaux à travers le projet de recherche-développement intitulé «Professionnalisation et renforcement de la compétitivité de la filière porc par la recherche-action en partenariat dans les départements de l’Ouémé et du Plateau au sud-est du Bénin ».

\section{REFERENCES}

Adeola A.C., Oseni S.O., Omitogun O.G., 2013. Morphological characterization of indigenous and crossbred pigs in rural and peri-urban areas of Southwestern Nigeria. Open J. Anim. Sci, 3 (3): 230-235, doi: 10.4236/ojas.2013.33034

Alenyorege B., Kodjo A.K., Addah W.A., 2015. Phenotypic characteristics of the Ashanti Black pig under intensive rearing. Vet. Sci., 5 (11): 22-27

Amills M., Ramirez O., Galman-Omitogun O., Clop A., 2013. Domestic pigs in Africa. Afr. Archaeol. Rev., 30 (1): 73-82, doi: 10.1007/s10437-012$9111-2$

Countrystat, 2017. Base de données statistiques. http://countrystat.org/home. aspx? $=$ BEN\&ta=053CPD050\&tr=7 (consulté 12 mai 2017)

Direction de l'élevage, 2015. Rapport annuel d'activité. Cotonou, Bénin $80 \mathrm{p}$.

FAO, 2012. Phenotypic characterization of animal genetic resources. Rome, Italy, 142 p.
Houndonougbo M.F., Adjolohoun S., Aboh B.A., Singbo A., Chrysostome C.A.A., 2012. Caractéristiques du système d'élevage porcin au sud-est du Bénin. Bull. Rech. Agron. Bénin, NS 2012 : 15-21

INSAE, 2015. Que retenir des effectifs de population en 2013 ? Cotonou, Bénin, $33 \mathrm{p}$.

Meyer C., 2016. Dictionnaire des sciences animales. Montpellier, France, Cirad, http://dico-sciences-animales.cirad.fr (consulté le 25 juin 2016)

Oluwole O.O., Tiamiyu A.K., Olorungbounmi T.O., Oladele-Bukola M.O., Akintoye N.A., 2014. Pre-weaning growth traits in Nigerian indigenous pig crossbreds. Agric. Sci., 5: 891-896, doi: 10.4236/as.2014.510096

Ramirez O., Ojeda A., Tomas A., Gallardo D., Huang L.S., Folch J.M.., Clop A. et al., 2009. Integrating Y-chromosome, mitochondrial, and autosomal data to analyze the origin of pig breeds. Mol. Biol. Evol., 26 (9): 2061 . 2072, doi: 10.1093/molbev/msp118

Youssao A.I., Koutinhouin G.B., Kpodekon T.M., Bonou A.G., Adjakpa A., Dotcho C.D.G., Atodjinou F.T.R., 2008. Pig production and indigenous genetic resources in suburban areas of Cotonou and Abomey-Calavi in Benin. Rev. Elev. Med. Vet. Pays Trop., 61 (3-4): 235-243, doi: 10.19182/ remvt.9995

Youssao A.K.I., Koutinhouin G.B., Kpodekon T.M., Yacoubou A., Bonou A.G., Adjakpa A., Ahounou S., Taiwo R., 2009. Amélioration génétique des performances zootechniques du porc local du Bénin par croisement avec le Large White. Int. J. Biol. Chem. Sci., 3 (4) : 653-662, doi : 10.4314/ijbcs. v3i4.47158

\section{Summary}

Youssao Abdou Karim I., Dotché I.O., Seibou Toleba S., Kassa K.S., Ahounou S.G., Salifou C., Dahouda M., Antoine-Moussiaux N., Dehoux J.-P., Mensah G.A. Phenotypic characterization of pig genetic resources in the departments of Oueme and Plateau in Benin

Pig farming is very common in Southern Benin and involves a diversity of breeds or populations. The aim of the study was to characterize the different pigs morphometrically and phenotypically. Thus, phenotypic data were collected from 149 pigs including 14 improved, 91 crossbred and 44 local pigs. Local pigs showed significantly lower morphological measurements $(p<0.05)$ than improved pigs and than pigs from crosses between improved and local pigs. Bristles of local pigs were significantly shorter $(p<0.05)$ than those of improved and crossbred pigs. The coat color varied from one genetic type to another. The most common color was uniform white, followed by uniform black in all genetic types. The head profile was straighter in local pigs, and more curved in improved and crossed pigs. Erect ears were less observed among crossbred pigs. Ears were front-facing in improved and crossbred pigs, whereas they were erect and rear-facing in local pigs. The presence of a corkscrew tail was significantly higher $(\mathrm{p}<$ 0.05 ) in improved pigs than in crossbred pigs, and it was higher in the latter one than in local pigs. A straight dorsal line was more often observed in local and crossbred pigs than in improved pigs where the line was more hollow. The crossbred pigs were very similar to the improved pigs.

Keywords: swine, phenotype, land race, crossbreeding, body measurement, Benin

\section{Resumen}

Youssao Abdou Karim I., Dotché I.O., Seibou Toleba S., Kassa K.S., Ahounou S.G., Salifou C., Dahouda M., Antoine-Moussiaux N., Dehoux J.-P., Mensah G.A. Caracterización fenotípica de los recursos genéticos porcinos en los departamentos de Ouémé y Plateau en Benin

La cría de cerdos es muy común en el sur de Benin e involucra una variedad de razas o poblaciones. El objetivo del estudio fue caracterizar los diferentes cerdos a los niveles morfométrico y fenotípico. Los datos fenotípicos se obtuvieron de 149 cerdos, incluyendo 14 cerdos mejorados, 91 cerdos cruzados y 44 cerdos locales. Los cerdos de tipo genético local mostraron medidas morfológicas significativamente menores $(p<0,05)$ que los cerdos mejorados y los animales cruzados entre cerdos mejorados y locales. Los pelos de cerdo local fueron significativamente más cortos $(p<0,05)$ que los de los cerdos mejorados y cruzados. El color de la piel varió de un tipo genético a otro. El color de la piel más común era el blanco uniforme, seguido del negro uniforme en todos tipos genéticos. El perfil de la cabeza era más sencillo en cerdos locales y más cóncavo en cerdos mejorados y cruzados. Las orejas erectas fueron menos observadas en los cruzados. Estas orejas estaban orientadas hacia adelante en cerdos mejorados y cruzados ya que estaban orientadas hacia atrás en cerdos locales. La proporción de cerdos mejorados con colas en forma de tirabuzón fue significativamente $(p<0,05)$ mayor que la en los cerdos cruzados, que sí mismo fue más alta que la en los cerdos locales. La línea dorsal recta fue más observada en los cerdos y cruces locales que en los cerdos mejorados en los que la línea era más curva. Los cerdos cruzados mostraron una gran similitud con los cerdos mejorados.

Palabras clave: cerdo, fenotipo, raza indígena, cruzamiento, medición del cuerpo, Benin 
